\title{
Facilitation based on inhibition
}

\author{
ROBERT A. RESCORLA \\ University of Pennsylvania, Philadelphia, Pennsylvania
}

\begin{abstract}
Three experiments were conducted using an autoshaping procedure in pigeons to examine the conditions under which facilitation develops to $B$ in an $A-\mid B A+$ paradigm. When $B$ is a diffuse stimulus and $A$ is a localized keylight, this paradigm results in $B$ 's facilitating pecking at $A$. Prior inhibitory conditioning of $A$ promoted the development of this facilitation more successfully than did prior excitatory or control treatment of $A$. These results suggest that facilitation develops especially well when an inhibitory stimulus is reinforced in the presence of the diffuse $B$. That outcome is consistent with the view that facilitation involves the removal of inhibition.
\end{abstract}

There has been considerable recent interest in Pavlovian conditioning paradigms in which one stimulus $(B)$ signals that another stimulus $(A)$ will be reinforced. One paradigm that has received particular attention is the "occasion setting" or "facilitation" paradigm, in which $A$ is nonreinforced when presented alone but reinforced when accompanied by $B$. In many such $A-\mid B A+$ procedures, $A$ becomes a conditioned excitor whereas $B$ remains relatively neutral and, instead, sets the occasion for (Holland, 1983) or facilitates (Rescorla, 1985) responding to $A$. That outcome is of interest because it suggests that $B$ plays a modulatory, rather than an elicitive, role in governing performance.

This facilitation paradigm is the procedural opposite of the more familiar $A+/ B A$ - conditioned-inhibition paradigm. In that paradigm, in which $A$ is reinforced except when $B$ is present, $A$ becomes an excitor and $B$ develops conditioned inhibition, interfering with the response to $A$. The fact that opposite procedures are used to establish conditioned facilitation and conditioned inhibition has encouraged the view that they are functionally opposite processes. According to that view, inhibitors and facilitators act in a comparable, but opposing, manner to modulate responding to excitors. Several results have encouraged the view that facilitators and inhibitors function as comparable opposites in both learning and performance. For instance, neither facilitators nor inhibitors commonly elicit a response of their own but, instead, require the presence of an excitator whose response they can modify. Moreover, the power of both stimuli is surprisingly resistant to simple Pavlovian reinforcement and extinction operations. However, facilitators and inhibitors readily change their properties when treated according to the other's paradigm (Rescorla, 1986a, 1987).

The view that facilitators and inhibitors are opposites has been further encouraged by the observation that facil-

This research was supported by National Science Foundation Grant BNS-8308176. Correspondence concerning this article should be sent to Robert A. Rescorla, Department of Psychology, University of Pennsylvania, 3815 Walnut Street, Philadelphia, PA 19104. tators are especially effective in promoting responding to excitors that have an inhibitory component. Rescorla (1985) observed facilitators to promote performance to excitors with a history of extinction, but not to similarly valued excitors lacking that history. Moreover, Rescorla (1987) found a facilitator to be especially effective with a compound stimulus with an inhibitory element. These results suggest that facilitators may act in part by reversing the action of conditioned inhibition.

The observation that inhibitory stimuli are especially effective targets for the performance effects of a facilitator suggests the possibility that they may also be especially effective targets for the establishment of facilitation. It may be the presence of $B$ at a time that an inhibitory $A$ is reinforced that allows $B$ to become a facilitator. Such a view would account for the observation that $B$ does not become a facilitator simply as a result of $B A+$ trials; it is necessary that $A-$ trials also be given. Those $A-$ trials presumably lead to extinction of $A$, possibly by an inhibitory process. As a result, $A$ has an inhibitory component when it is reinforced in $B$ 's presence. Paradoxically, this view might also explain the finding (Rescorla, 1986b) that pretraining $A$ as an excitor promotes $B$ 's facilitation when it is trained in an $A-\mid B A+$ paradigm. Pretraining of excitation to $A$ might make the $A$ - trials especially effective in establishing inhibition.

If this line of reasoning has merit, then $B$ should become a particularly strong facilitator if it is trained in an $A-/ B A+$ paradigm with an inhibitory $A$. The three experiments reported here explored this possibility. All three experiments used pigeon subjects in an autoshaping procedure in which a keylight $A$ was paired with food on some trials. Diffuse auditory and visual stimuli served as the facilitators. Under those conditions, birds come to peck $A$ primarily in the presence of the diffuse facilitators (see, e.g., Rescorla, 1985). Experiments 1 and 2 compared inhibitory and neutral stimuli in the role of $A$; Experiment 3 compared inhibitory and excitatory stimuli in that role. In all experiments, the facilitators were tested for their ability to promote responding to a common transfer keylight. 


\section{EXPERIMENT 1}

This experiment directly compared an inhibitory and a neutral keylight as targets for establishing facilitation to a diffuse stimulus. Each bird was exposed to two concurrent $A-\mid B A+$ paradigms in which a neutral or an inhibitory keylight played the role of $A$ and a diffuse auditory or visual stimulus served as $B$. Because the two $A$ stimuli could be expected to control different levels of responding, the facilitation developed by the $B$ stimuli was assessed against a common transfer target keylight. Both $B$ stimuli were tested for their ability to facilitate responding to a trained and partially extinguished excitor.

One other feature of this experiment should be noted. Prior to their facilitation training, both $B$ stimuli were given simple excitatory conditioning by repeated pairings with food. This was done because previous experiments (Rescorla, 1986b) had found such pretraining to slow the development of facilitation and hence to expose especially well the impact of excitatory pretraining of $\boldsymbol{A}$. It was hoped that this pretraining of the diffuse stimuli would also enhance the impact of inhibitory pretraining of $A$.

\section{Method}

Subjects and Apparatus. The subjects were 16 experimentally naive female Carneau pigeons about 1 year old. They were housed in pairs and maintained at $75 \%$ of their free-feeding weights.

The apparatus had eight identical operant chambers, each measuring $27 \times 27 \times 35 \mathrm{~cm}$. The metal front panel of each chamber had a $5 \times 5 \mathrm{~cm}$ food magazine in its center, located $5 \mathrm{~cm}$ above the wiremesh floor. Three response keys, $2.5 \mathrm{~cm}$ in diameter, were located one directly above the hopper and one on either side of the center of the front wall, $20 \mathrm{~cm}$ above the floor. Located behind the lefthand key was an IEE in-line projector that permitted the transillumination of the key with three light stimuli, red (R), green (G), and an $X$. The red and green lights were confined to the left-hand portion of the response key; the remainder of the key remained dark. The $X$ consisted of two 1-mm black lines crossing at the center of an otherwise white background. These stimuli were generated by Ektachrome slides of drawings composed of "Color-aid" artist's paper.

The other three walls and ceiling of the chambers were composed of clear Plexiglas. These chambers were placed in sound- and lightattenuating shells with ventilation fans providing background noise of $62 \mathrm{~dB}$ re $20 \mu \mathrm{N} / \mathrm{m}$. On the rear wall of those shells was mounted a 6-W bulb that was continuously illuminated during the session, except when it was interrupted at a rate of $1 / \mathrm{sec}$ to provide a diffuse visual signal $(L)$. The houselight also was turned off during the operation of the food hopper. That hopper contained Purina Pigeon Grain. Also mounted on the rear wall of the isolation chamber was a speaker that permitted the presentation of an $80-\mathrm{dB}$ white noise $(\mathrm{N})$.

Procedure. The birds were initially given 3 days of magazine training. On Day 1, they were placed in the chambers with the food hoppers raised to permit access to grain. After they had consumed grain for approximately $15 \mathrm{sec}$, the hoppers were lowered briefly. Then the time of grain availability was gradually shortened and the time between hopper operations lengthened until the birds reliably ate with 5-sec hopper operations spaced approximately $1 \mathrm{~min}$ apart. On each of the next 2 days, the birds received 445 -sec hopper operations spaced a mean of 1 min apart.

Over the next 9 days, all birds received initial autoshaping with the $X$ stimulus. On each day, they received 485 -sec presentations of $X$, each terminating in 5 sec of grain ( + ). In this and all subse- quent phases of the experiment, the intertrial interval (ITI) was variable around a mean of $1 \mathrm{~min}$.

On each of the next 20 days, the birds received a treatment designed to make one colored keylight a conditioned inhibitor while leaving the other relatively neutral. Half of the birds received 12 presentations of each of three trial types $\mathrm{X}+, \mathrm{RX}-$, and $\mathrm{G}-$. The other half of the birds received the same treatment with $R$ and $G$ interchanged. All stimulus presentations were $5 \mathrm{sec}$ long; on compound trials, the color immediately preceded $X$.

On each of the next 5 days, the birds received Pavlovian conditioning of L and N. On each day, all birds received 1815 -sec reinforced presentations each of $L$ and $N$. To assure stable differences in the conditioning of $R$ and $G$ at the beginning of facilitation training, conditioned-inhibition training was reinstituted on the next 5 days. At the end of the last of these sessions, all birds received two test presentations each of X, RX, and GX, in order to verify that conditioned-inhibition training had, indeed, endowed the colors with differential ability to inhibit responding to $X$.

On each of the next 6 days, all birds were exposed to two concurrent facilitation paradigms, using $R$ and $G$ to condition facilitation to $\mathrm{L}$ and $\mathrm{N}$. Half of the birds received 12 trials each of $\mathrm{LR}+$, R-, NG+ and G-. The other half received the same treatment except that the roles of $\mathbf{R}$ and $\mathbf{G}$ were interchanged. Treatments were arranged to be orthogonal to those of the preceding stage, so that for half the animals $\mathrm{L}$ was reinforced in conjunction with an inhibitor and $\mathrm{N}$ in conjunction with a neutral stimulus; for the other half, $\mathrm{L}$ was reinforced in conjunction with a neutral stimulus and $\mathbf{N}$ in conjunction with an inhibitor. All keylight presentations were $5 \mathrm{sec}$ long; on compound trials, the 15-sec diffuse stimulus was initiated $10 \mathrm{sec}$ prior to the keylight illumination.

Over the next 2 days, responding to $X$ was extinguished, in order to make it an appropriate target against which to test the facilitatory power of $\mathrm{L}$ and $\mathrm{N}$. On each day, the birds received 36 nonreinforced 5-sec presentations of $\mathrm{X}$.

A single test session was given on the next day. That session contained 3 nonreinforced presentations each of X, LX, and NX. The $X$ continued to be $5 \mathrm{sec}$ long; $\mathrm{L}$ and $\mathrm{N}$ were $15 \mathrm{sec}$ long and began $10 \mathrm{sec}$ prior to the onset of $\mathrm{X}$. The question of primary interest was whether the diffuse stimulus trained in conjunction with the inhibitory color would be more successful at promoting responding to $X$.

\section{Results and Discussion}

Initial autoshaping of $\mathrm{X}$ resulted in a terminal level of 64 responses/min. As shown in Figure 1, conditioning of inhibition to the colors proceeded with a pattern characteristic of such sequential conditioned-inhibition paradigms. Early in training, the color that preceded nonreinforced presentations of $\mathrm{X}$ (here labeled $\mathrm{I}$, for inhibitor) came to produce considerable pecking, as a result of second-order conditioning. However, with further training, responding to I alone extinguished. Similarly, early in training, the excitor evoked more responding when it was preceded by I (labeled $\mathrm{E}-$ ) than when it was presented alone $(E+)$; later in training, I had an inhibitory effect on responding to $\mathrm{X}$. There was little responding at any point to the other color (here labeled $C$, for control). On the final day of conditioned-inhibition retraining, no responding occurred to either I or $\mathrm{C}$ presented alone. The mean response rates to $X$ were 77 and 32 when it was presented alone and preceded by $I$, respectively [Wilcoxon $T(16)=5, p<.01]$. That the ability of I to inhibit responding to $X$ depended on its training history was veri- 


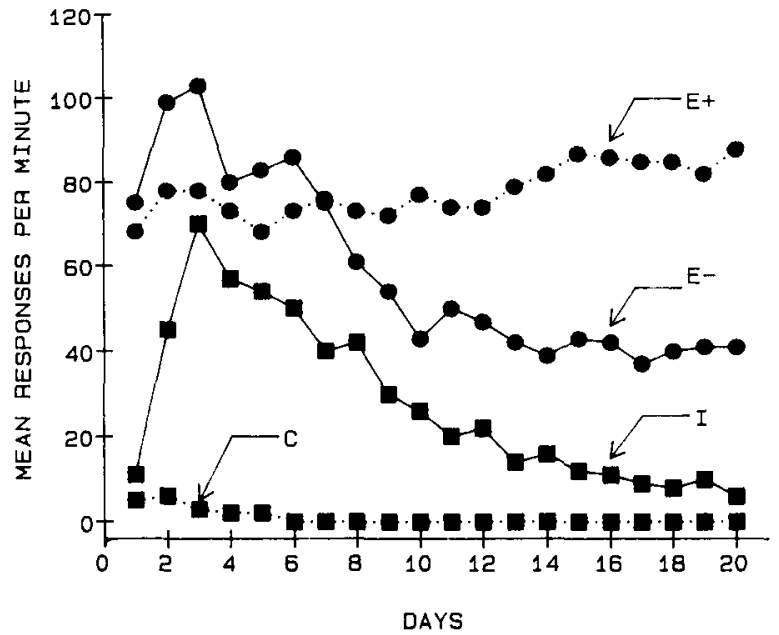

Figure 1. Development of sequential conditioned inhibition in Experiment 1. One keylight was reinforced when presented alone $(E+)$ but not $(E-)$ when preceded by the inhibitor $(\mathbf{I})$. The $C$ keylight was presented without reinforcement throughout.

fied in the test session at the end of the final day of conditioned-inhibition training. During that test, the mean response rates to $\mathrm{X}$ when it was presented alone, preceded by $I$, and preceded by $C$ were 70,32 , and 78 , respectively. A Wilcoxon test showed responding to $\mathrm{X}$ preceded by I to be reliably below that to each of the other two cases $\left[T_{\mathrm{s}}(15)=0,0 ; p \mathrm{~s}<.01\right]$.

The left-hand portion of Figure 2 shows the course of facilitation training, plotted separately for the diffuse stimulus being trained with I and with $\mathrm{C}$. Because responding did not differ as a function of the identity of the facilitator, the results are not shown separately for $\mathrm{L}$ and $\mathrm{N}$. It is clear that substantial responding occurred only when I was compounded with its diffuse stimulus. Responding seldom occurred to I alone, or to $C$ either when it was presented alone or in conjunction with its diffuse stimulus. On the final day of training, the responding to I was reliably greater during its facilitator than when it was presented alone $[T(11)=0, p<.01]$.

The right-hand side of Figure 2 shows the data of most interest, from the test presentations of $\mathrm{X}$ alone and in conjunction with each facilitator. Responding was quite low to $\mathrm{X}$ alone, and each facilitator augmented that responding. However, that augmentation was greater for the diffuse stimulus that had been trained in conjunction with an inhibitor. Responding to $X$ during that facilitator was reliably greater than that either to $X$ alone $[T(13)=2$, $p<.01]$ or to $\mathrm{X}$ in conjunction with the facilitator trained with $C[T(12)=10.5, p<.05]$, but those two did not differ.

The results of training with a neutral target replicate those reported by Rescorla (1986b). Little facilitation developed to a diffuse stimulus that had received excitatory pretraining prior to facilitation training with a neutral target. However, considerable facilitation was conditioned to $B$ in an $A-B A+$ paradigm when $A$ had been pretrained as an inhibitor. That data pattern was observed both when the facilitators were tested in conjunction with their training targets and when they were tested against a common transfer keylight. These results suggest that an inhibitor is a superior target for facilitation training.

However, the present results must be interpreted with caution because of the particular training history of the
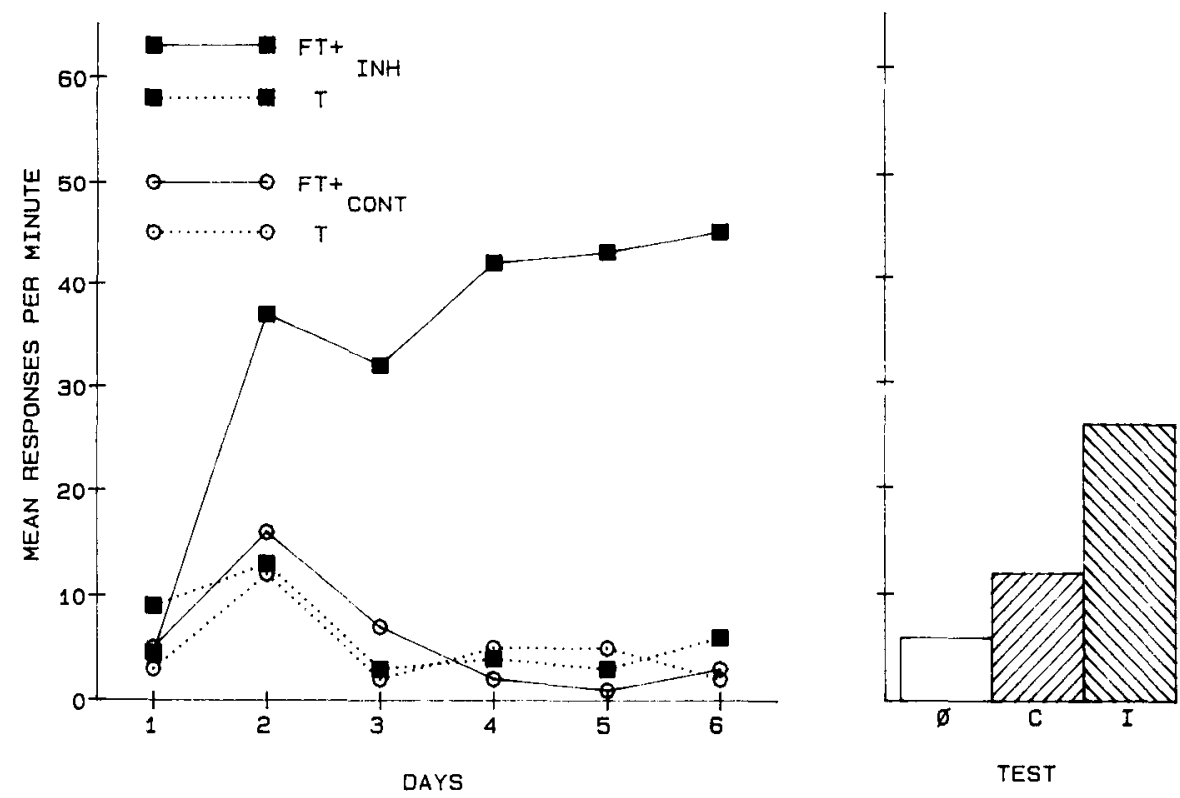

Figure 2. Development and testing of facilitation in Experiment 1. The left panel shows responding to inhibitory and control target stimuli ( $T$ ) when presented alone without reinforcement or in the presence of a diffuse facilitator (FT) with reinforcement. The right panel shows responding to an extinguished key presented alone ( $(0)$ and in conjunction with the facilitator trained with a control (C) target and an inhibitory (I) target. 
conditioned inhibitor. Because a sequential procedure was used to train inhibition, I differed from $\mathrm{C}$ not only in its terminal inhibitory properties but also in its history of excitatory properties. It seems possible that it was this history of excitation that made I a superior target for establishing facilitation. This possibility must be taken particularly seriously because Rescorla (1986b) found excitatory targets quite potent in promoting facilitation. Although the I stimulus no longer had net excitatory strength by the time it was used in facilitation training, its history of such strength may have been sufficient to make it a powerful base upon which to establish facilitation.

\section{EXPERIMENT 2}

This experiment had two intentions. The first was to replicate the finding of Experiment 1, that inhibitory targets promote better facilitation. The second was to do so with an inhibitor lacking a prior history of excitation. This was accomplished by training the inhibitor with a simultaneous, rather than a sequential, conditioned-inhibition paradigm. When the inhibitor and excitor are presented simultaneously on the nonreinforced $B A$ trials, initial excitatory conditioning of $B$ is minimized but its inhibition nevertheless develops fully.

\section{Method}

Subjects and Apparatus. The subjects were 16 experimentally naive female Carneau pigeons about 1 year old. They were maintained as in Experiment 1. The apparatus was that of Experiment 1, arranged to permit the presentation of two color stimuli, red $(R)$ and green $(G)$, as well as a pattern of vertical 1-mm black stripes spaced $1 \mathrm{~mm}$ apart on a white background (V). The colored stimuli could be projected on the left-hand side of the response key, and the $V$ appeared on the right-hand side. When any stimulus was presented alone, the other half of the key remained black. When compounds were presented, the two sides were separated by a 1-mm black vertical line.

Procedure. The birds were magazine trained as in Experiment 1. They then received initial autoshaping with $V$ over 6 days. On each day, they received 485 -sec presentations of $V$, each ending in $5 \mathrm{sec}$ of grain. In this and all subsequent phases of the experiment, the ITI was variable around a mean of $1 \mathrm{~min}$.

Conditioned-inhibition training was conducted on each of the next 10 days. Half of the birds received 12 trials each of $\mathrm{V}+, \mathrm{GV}-$, and $\mathrm{R}-$; the remaining half of the birds received the same training with the roles of $R$ and $G$ interchanged. All stimulus presentatons were $5 \mathrm{sec}$ long, and the color occurred simultaneously with $V$ on compound trials.

On each of the next 5 days, the birds received Pavlovian conditioning of $L$ and $N$. Each day contained 1815 -sec reinforced presentations each of $\mathrm{L}$ and $\mathrm{N}$. Conditioned-inhibition training resumed for the next 5 days. At the end of Day 5 of conditioned-inhibition training, a brief test was given to assess the success with which the two colors had been given different values. All birds received two nonreinforced presentations each of $\mathrm{V}, \mathrm{GV}$, and RV.

On each of the next 12 days, all birds were given two concurrent facilitation paradigms. On each day, half the animals received 12 presentations each of $\mathrm{LR}+, \mathbf{R}-, \mathbf{N G}+$, and $\mathbf{G}-$. The remaining half of the animals received the same training, except that $R$ and $G$ were interchanged. The treatments were counterbalanced so that for half the animals $\mathrm{L}$ was reinforced in conjunction with an inhibitory color and $\mathrm{N}$ in conjunction with a neutral color, whereas for the remain- ing half of the animals the roles of $\mathrm{L}$ and $\mathrm{N}$ were interchanged. The $R$ and $G$ keylights were $5 \mathrm{sec}$ long; $\mathrm{L}$ and $\mathrm{N}$ were $15 \mathrm{sec}$ long and began $10 \mathrm{sec}$ prior to the keylight on compound trials.

On each of the next 2 days, the responding to $V$ was extinguished. Each day contained 36 nonreinforced presentations of $V$.

A single test session was given on the next day. That session began with 12 nonreinforced presentations of $V$ and continued with 3 nonreinforced presentations each of V, LV, and NV. The question of interest was whether the diffuse stimulus trained in conjunction with the inhibitory color would more successfully facilitate responding to $\mathrm{V}$.

\section{Results and Discussion}

Conditioned inhibition developed with a pattern characteristic of training with simultaneous compounds. Responding remained high to reinforced $\mathrm{V}$ presentations and low to the nonreinforced color presentations, throughout training. By contrast, responding was initially high to the simultaneous compound of $\mathrm{V}$ with a color but rapidly declined over training. On Day 1 of training, the mean response rates to the excitor alone, the excitor in compound with the (eventual) inhibitor, and the control stimulus were 101, 96, and 7 responses/min, respectively. Responding to the simultaneous compound declined rapidly to less than 20/min on Day 6 of training. Although simultaneous presentation does not provide direct evidence on possible excitatory conditioning of the color, there was no indication that responding to the compound ever exceeded that to $\mathrm{V}$ alone. On the final day of training, the mean response rates were 92,0 , and 0 to the excitor, the compound, and the control stimulus, respectively.

Responding during the brief test at the end of conditioned inhibition training verified that the inhibitory and control colors had acquired differential abilities to interfere with responding to $\mathrm{V}$. During that test, the mean response rate to $\mathrm{V}$ alone was 88 whereas that to $\mathrm{V}$ in compound with the inhibitory color was 2 and in compound with the control color was 68 . A Wilcoxon test found the response rate to be reliably lower during the compound containing the inhibitor as compared both with $\mathrm{V}$ alone and with the compound containing the control color $[T s(15)=0, p s<.01]$. The suppression produced by the control color fell short of significance $[T(13)=27]$.

The left-hand side of Figure 3 shows the course of facilitation training of $\mathrm{L}$ and $\mathrm{N}$. The results are separated according to whether the target keylight was inhibitory or neutral, but collapsed across the identities of the facilitators, which showed similar responding. It is clear that substantial responding developed only during the compound of a facilitator and an inhibitory target. By the end of training, responding was greater to the inhibitory target when it was presented with its facilitator than when it was presented alone $[T(12)=7, p<.05]$. On the other hand, the other facilitator did not produce a reliable augmentation of responding to the neutral target.

The right-hand side of Figure 3 shows the results of most interest, those from the transfer test of the facilitators with V. During that test, responding was low to the extinguished $\mathrm{V}$ when it was presented alone; however, the facilitator trained with an inhibitor augmented respond- 

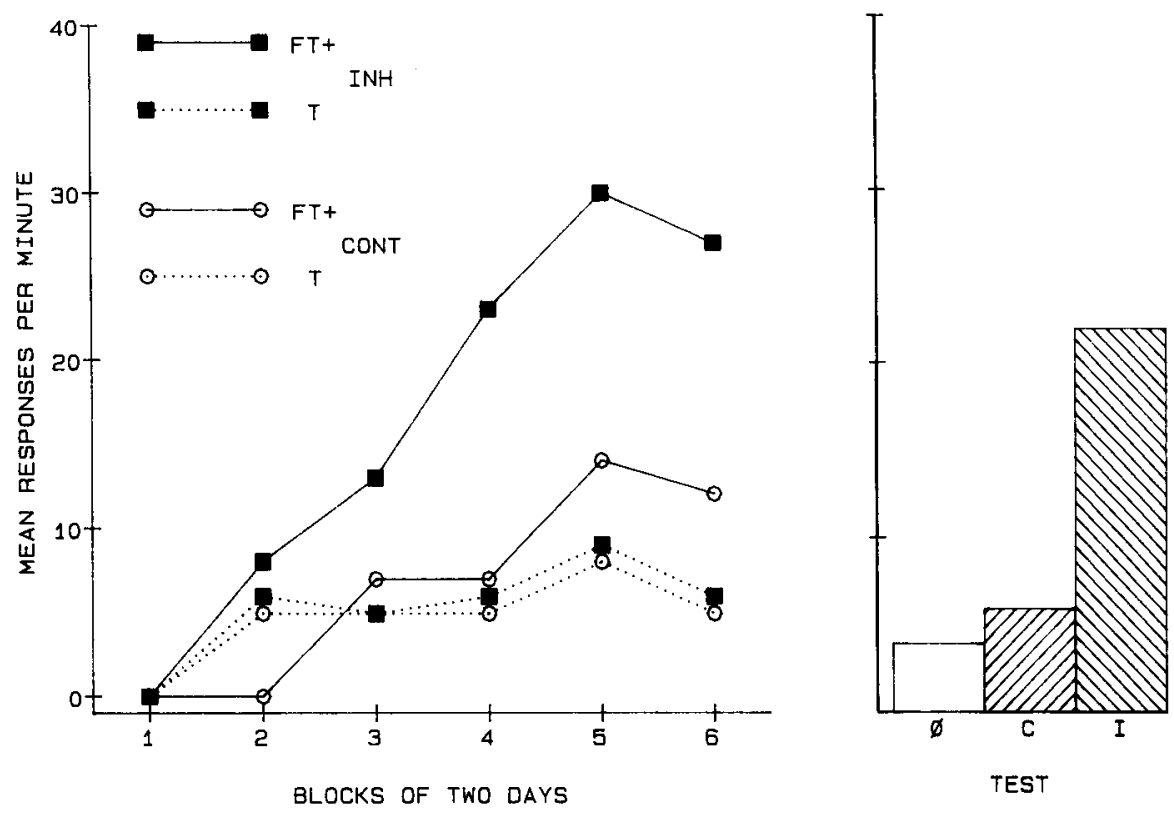

Figure 3. Development and testing of facilitation in Experiment 2. Designations are as in Figure 2.

ing to $\mathrm{V}[T(11)=0, p<.01]$. Responding to the compound of $V$ and the facilitator trained with a neutral target did not differ from that to $\mathrm{V}$ alone but was below that to $\mathrm{V}$ presented together with the inhibitory-trained facilitator $[T(10)=1, p<.01]$.

These results confirm those of Experiment 1. A facilitator trained in conjunction with a conditioned inhibitor was more able to promote responding to a transfer stimulus than was one trained in conjunction with a neutral stimulus. Moreover, in the present experiment there was no reason to suspect that the inhibitor had passed through an initial excitatory phase. Indeed, the target stimulus showed only weak signs of ever developing excitation. That makes less plausible the interpretation of Experiment 1 in terms of the importance of an excitatory history for the target keylight.

\section{EXPERIMENT 3}

This experiment had two goals. First, it sought to compare directly inhibitory and excitatory stimuli as targets on the basis of which to establish facilitation. As noted earlier, Rescorla (1986b) reported that excitatory keylights are especially effective targets for establishing facilitation. One interpretation of that finding is that targets with excitatory pretraining are particulary rapid at acquiring conditioned inhibition on the $A-$ trials of an $A-\mid B A+$ paradigm. Consequently, their success may result not from their excitatory pretraining but rather from the fact that pretraining promotes the development of inhibition, which, in turn, assists the training of facilitation. If that interpretation is correct, inhibitory pretraining should be a more direct way of promoting facilitation; that is, inhibitory stimuli should be more successful than equally strong excitors at encouraging facilitation. Accordingly, in the present experiment, excitatory and inhibitory targets of equal, but opposite, strength were compared for their ability to establish facilitation.

The second goal of this experiment was to explore the necessity of excitatory pretraining of the diffuse stimuli for observing the results reported here. This pretraining was used as a technical device because it is known to slow the development of facilitation and might therefore better expose the influence of other variables. However, it is also possible that the special advantage of an inhibitory target may be observed only with facilitators that have received excitatory pretraining. For instance, it may be that an inhibitory target is capable of undermining the excitation controlled by the diffuse stimulus. Because excitatory pretraining of a diffuse stimulus is known to slow facilitation, a stimulus that undermines that pretraining might produce a relative speeding of facilitation. Consequently, in the present experiment, the diffuse stimuli were preexposed without reinforcement prior to their training in facilitation.

In other regards, the procedure of Experiment 3 was similar to those of Experiments 1 and 2. Birds received excitatory pretraining with one keylight and inhibitory pretraining with another. Training was conducted until the inhibitor was sufficiently strong to almost entirely prevent responding to the excitor. Those keylights then served the role of $A$ in two concurrent $A-B B A+$ paradigms; $\mathrm{L}$ and $\mathrm{N}$, each with a history of nonreinforcement, served in the role of $B$. Then $\mathrm{L}$ and $\mathrm{N}$ were each examined for their ability to promote responding to a common third keylight.

\section{Method}

Subjects and Apparatus. The subjects were 16 female Carneau pigeons about 1 year old. They had participated in another autoshap- 
ing experiment which had used different stimuli and a different response key. They had had no prior experience with the sort of conditional discriminations used here. The assignment of birds to groups in the present experiment was random with respect to their previous treatments. They were housed in pairs and maintained at $75 \%$ of their free-feeding weights. The apparatus was that of Experiment 2 . In the present experiment, the response key could be illuminated with the $R, G$, and $V$ of the previous experiments, as well as by a grid pattern like $\mathrm{V}$ but oriented along the horizontal dimension $(\mathrm{H})$

Procedure. Because these birds had participated in a previous experiment, no magazine training was necessary. During each of the first four sessions, the birds received conditioning designed to establish either $\mathbf{R}$ or $\mathrm{V}$ as a conditioned excitor. Half of the birds received $365-\mathrm{sec}$ reinforced presentations of $R$ in each session; the other half received 365 -sec reinforced presentations of $V$.

Conditioned-inhibition training was conducted on each of the next 12 days. Each session contained 12 reinforced presentations of the excitatory stimulus from the previous days. In addition, all birds received 12 nonreinforced presentations of the 5-sec RV compound. As a result of this training, $R$ was excitatory and $V$ inhibitory for half the birds and $V$ was excitatory and $R$ inhibitory for the other half.

On each of the next 2 days, the birds received nonreinforced presentations of the $\mathrm{L}$ and $\mathrm{N}$ diffuse stimuli later to be made facilitators. Each session contained 1815 -sec presentations of each stimulus. Conditioned-inhibition training was resumed for the next 4 days to ensure stable differences between $R$ and $V$ when that facilitation training began.

Facilitation training was conducted on each of the next 4 days. Half of the birds in each of the preceding two groups received 12 trials each of $\mathrm{LR}+, \mathrm{R}-, \mathrm{NV}+$, and $\mathrm{V}-$. The remaining half of the birds received the same treatment, with the roles of $R$ and $V$ interchanged. The result was that for half of the birds, $L$ was trained in conjunction with an excitatory target and $\mathrm{N}$ was trained with an inhibitory target. For the remaining half of the birds, $L$ was trained with an inhibitory target and $\mathrm{N}$ with an excitatory target. As in the previous experiments, $\mathrm{L}$ and $\mathrm{N}$ were $15 \mathrm{sec}$ long and began $10 \mathrm{sec}$ prior to the keylights.

Over the next 4 days, a compound of green and horizontal stripes (GH) was prepared as a target against which to assess the facilitators. On the first day, all birds received 24 reinforced presentations of the $\mathrm{GH}$ compound. On each of the next 3 days, they received 24 nonreinforced presentations of $\mathrm{GH}$.

The next day contained a single test session. During the first half of that session, the birds received six presentations of each of the four types of trials they had received during facilitation training. They then received four nonreinforced presentations of $\mathrm{GH}$ alone and four each of $\mathrm{GH}$ in compound with $\mathrm{L}$ and with $\mathrm{N}$. The question of interest was whether $\mathrm{L}$ and $\mathrm{N}$ would be differentially successful at promoting responding to the common $\mathrm{GH}$ as a function of the excitatory or inhibitory value of the keylight with which they had been trained.

\section{Results and Discussion}

Conditioned inhibition training proceeded without incident. By the end of training, the mean response rate to the excitor was 167.2 , whereas that to the RV compound was 4.1. The $R$ and $V$ excitatory stimuli did not differ in response rate. No responding was observed at all during the $\mathrm{L}$ and $\mathrm{N}$ stimuli when they were presented without reinforcement.

The left-hand side of Figure 4 shows the course of facilitation training. Responding developed rapidly to both keylights presented alone and in compound with the potential facilitators. This rapid development of responding is characteristic of facilitation designs in which the diffuse stimuli have not received excitatory pretraining (e.g., Rescorla, 1986b). However, with continued training, responding remained high to both compounds of diffuse stimulus and keylight and fell away to both keylights
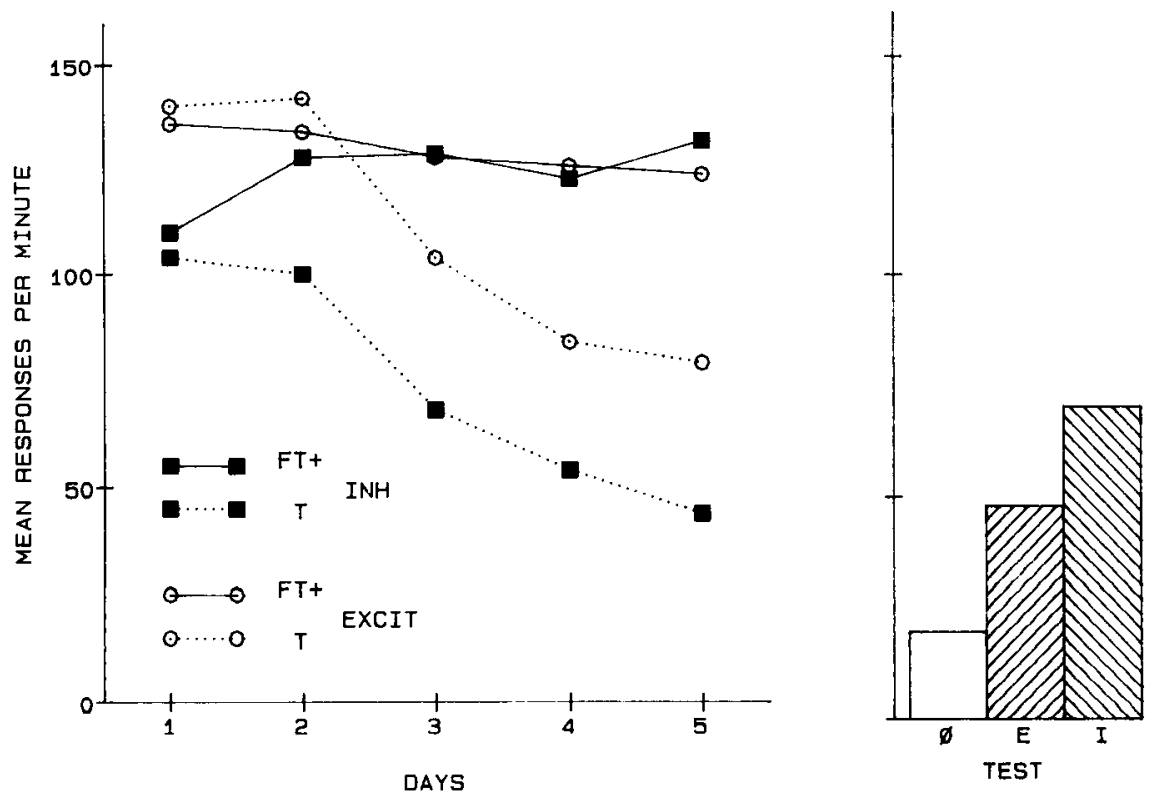

Figure 4. Development and testing of facilitation in Experiment 3. The left panel shows responding to inhibitory and excitatory target stimuli when presented alone $(T)$ without reinforcement or in the presence of a diffuse facilitator (FT) with reinforcement. The right panel shows responding to an extinguished keylight presented alone $(\theta)$ and in conjunction with the facilitator trained with an excitatory (E) target and an inhibitory (I) target. 
presented alone. By the final day of training, responding was greater in the presence of a facilitator than in its absence, for both the excitatory and inhibitory targets [Ts $(16)$ $=27,7 ; p \mathrm{~s}<.05]$. However, facilitation was greater with the inhibitory target, a difference that was exhibited primarily on target-alone trials. When the keylights were presented alone, there was more responding to the excitatory keylight than to the inhibitory keylight $[T(16)=$ $23, p<.05]$. Responding to the two keylights was similar in the presence of their facilitators.

The right-hand side of Figure 4 shows the results of the test session during which the GH stimulus was presented alone and in conjunction with each facilitator. Responding was low to $\mathrm{GH}$ alone, but was augmented by the presence of either facilitator. However, the amount of that augmentation was greater for the facilitator that had been trained in conjunction with an inhibitory keylight. Responding to $\mathrm{GH}$ was greater in the presence of the inhibitory-trained facilitator than when it was presented alone $[T(15)=0, p<.01]$. Responding in the presence of the excitatory-trained facilitator was greater than that to $\mathrm{GH}$ alone $[T(15)=0, p<.01]$ but less than in the presence of the inhibitory-trained facilitator $[T(13)$ $=5.5, p<.011$.

These results are in agreement with those reported here and previously. Pretraining a target stimulus as either an excitor or an inhibitor apparently enhances its ability to train facilitation. However, inhibitory pretraining is the more successful of the two procedures.

The superiority of inhibition as a pretraining procedure for a target stimulus makes less attractive an interpretation in terms of the inhibitor's having some unobserved excitatory association that is responsible for its impact. Experiments 2 and 3 both used a simultaneous procedure for establishing the inhibitor in order to minimize the possibility that the inhibitor would have initial excitatory strength. But other results imply that even under those circumstances, the inhibitor may develop some excitation by virtue of its within-compound association with the excitor (e.g., Cunningham, 1981; Speers, Gillan, \& Rescorla, 1980; Williams, Travis, \& Overmier, 1986). However, whatever the excitatory contribution of such associations to the present inhibitor, they are unlikely to be stronger than that directly conditioned to the excitor. Indeed, the greater facilitation based on an inhibitory target suggests, to the contrary, that the excitor serves as a target by virtue of its inhibition.

\section{GENERAL DISCUSSION}

These results support the view that a conditioned inhibitor is an especially good target upon which to build conditioned facilitation. It may be that the general condition for producing a facilitator is that a stimulus be present when a target with an inhibitory component is reinforced. In the typical $A-\mid B A+$ facilitation paradigm, $A$ initially acquires some excitatory conditioning that extinguishes on the $A$-trials. That provides the opportunity for $B$ to be present when that inhibitory $A$ is then reinforced. Treatments that prevent $A$ from becoming initially excitatory, such as excitatory pretraining on $B$, would also prevent $A$ from developing inhibition on the $A-$ trials. As anticipated, those treatments also prevent $B$ from developing into a facilitator (Rescorla, 1986b). Similarly, procedures that enhance the initial excitatory strength of $A$, thereby especially encouraging the development of inhibition in the $A$ - trials, enhance the facilitation conditioned to $\mathrm{B}$. The present experiments suggest that directly making $A$ an inhibitor can enhance $B$ 's facilitation even in the absence of excitatory training of $A$. The superiority of inhibitory over excitatory pretraining supports the view that $A$ 's inhibition is its critical property.

It is of interest to note that the conditions identified here as promoting facilitation are the same as those that have been described elsewhere as producing superconditioning of excitation to $B$. In agreement with the predictions of the Rescorla-Wagner model, several authors have reported that a stimulus reinforced in the presence of an inhibitor becomes especially excitatory (Rescorla, 1971; Wagner, 1971). But it seems unlikely that the present observations of enhanced facilitation simply reflect enhanced excitatory conditioning. Previous results (e.g., Rescorla, $1985,1986 a, 1986 \mathrm{~b}, 1987)$ suggest that a facilitator's action is relatively independent of its simple excitatory strength. Diffuse stimuli trained as excitors do not take on the ability to promote responding to a keylight target. Moreover, the acquisition of facilitation appears to be hindered for stimuli that are already conditioned excitors. On the other hand, it is possible that the results previously identified as demonstrating superconditioning involve facilitation. The facilitation developed by a stimulus reinforced in the presence of an inhibitor may allow it to promote responding to situational cues that have otherwise undergone extinction. In many conditioning preparations, responding during a stimulus would have a similar form whether it is attributable to that stimulus or to the background. Consequently, prior reports of superconditioning of a CS might, instead, be interpreted as superior facilitation training that allows the stimulus to promote responding to an ambiguous situational stimulus.

The present results are consistent with a view of Pavlovian conditioning according to which CSs develop excitatory associations with the US whereas inhibitors and facilitators modulate the operation of those associations. For instance, inhibitors and facilitators may act by changing the threshold of the US representation that the CS is attempting to activate by way of its excitatory association. That raised threshold may make reinforcement especially effective for establishing a facilitator. Indeed, a facilitator present during that reinforcement might then acquire the ability to lower the threshold. Moreover, it 
might transfer that ability to other stimuli that have artificially raised thresholds, as has been observed here and previously (e.g., Rescorla, 1985, 1986b).

If this view is correct, the modulatory functions of facilitators and inhibitors are as important as the excitatory functions of a CS in promoting behavior. Although the various types of stimuli may involve associations with the same US, they function in quite different ways. It is then important to understand not only how they function, but also how they acquire those functions.

\section{REFERENCES}

Cunningham, C. L. (1981). Associations between the elements of a bivalent compound stimulus. Journal of Experimental Psychology: Animal Behavior Processes, 7, 425-436.

Holland, P. C. (1983). "Occasion-setting" in Pavlovian feature positive discriminations. In M. L. Commons, R. J. Herrnstein, \& A. R. Wagner (Eds.), Quantitative analyses of behavior: Vol. IV. Discrimination processes (pp. 183-206). Cambridge, MA: Ballinger.

Rescorla, R. A. (1971). Variation in the effectiveness of reinforcement and nonreinforcement following prior inhibitory conditioning. Learning \& Motivation, 2, 113-123.
Rescorla, R. A. (1985). Conditioned inhibition and facilitation. In R. R. Miller \& N. S. Spear (Eds.), Information processing in animals: Conditioned inhibition (pp. 299-326). Hillsdale, NJ: Erlbaum.

RESCORLA, R. A. (1986a). Extinction of facilitation. Journal of Experimental Psychology: Animal Behavior Processes, 12, 16-24.

Rescorla, R. A. (1986b). Facilitation and excitation. Journal of Experimental Psychology: Animal Behavior Processes, 12, 325-332.

Rescorla, R. A. (1987). Facilitation and inhibition. Journal of Experimental Psychology: Animal Behavior Processes, 13, 250-259.

Speers, M. A., Gillan, D. J., \& Rescorla, R. A. (1980). Withincompound associations in a variety of compound conditioning procedures. Learning \& Motivation, 11, 135-149.

W AGNER, A. R. (1971). Elementary associations. In H. H. Kendler \& J. T. Spence (Eds.), Essays in neobehaviorism: A memorial volume to Kenneth W. Spence (pp. 187-213). New York: Appleton-CenturyCrofts.

Williams, D. A., Travis, G. M., Overmier, J. B. (1986). Withincompound associations modulate the relative effectiveness of differential and Pavlovian conditioned inhibition procedures. Journal of $E x$ perimental Psychology: Animal Behavior Processes, 12, 351-362.

(Manuscript received May 28, 1987; revision accepted for publication November 23, 1987.) 